\title{
Oscillations in the wings of sunspot transition region lines
}

\author{
N. Brynildsen ${ }^{1}$, P. Maltby ${ }^{1}$, O. Kjeldseth-Moe ${ }^{1}$, and K. Wilhelm² \\ 1 Institute of Theoretical Astrophysics, University of Oslo, PO Box 1029 Blindern, 0315 Oslo, Norway \\ e-mail: nils. brynildsen@astro.uio.no;per .maltby@astro.uio.no;olav.kjeldseth-moe@astro.uio.no \\ 2 Max-Planck-Institut für Aeronomie, 37191 Katlenburg-Lindau, Germany \\ e-mail: wilhelm@linmpi.mpg.de
}

Received 4 November 2002 / Accepted 15 December 2002

\begin{abstract}
We explore a new approach to the investigation of optically thin emission lines by comparing the intensity variations in opposite wings of the spectral lines and apply the method to investigate the oscillations above sunspots. The observations show that it is easier to detect the 3 min oscillations above sunspots in the short wavelength ("blue") wing than in the long wavelength ("red") wing of the lines. The observed oscillations are compatible with the spectral line signatures of upwardly propagating acoustic waves.
\end{abstract}

Key words. Sun: sunspot oscillations - Sun: transition region - EUV radiation - line wing

\section{Introduction}

The detection of umbral flashes in the sunspot chromosphere (Beckers \& Tallant 1969) fostered the idea that crucial information about the physics of sunspots could be revealed by investigating the oscillations in the sunspot atmosphere. The oscillatory power shows a dominant peak in the 3 min band from the cold regions where the $\mathrm{CO}$ vibration-rotation band at $4.66 \mu \mathrm{m}$ originates (Solanki et al. 1996), through the sunspot chromosphere (e.g., Lites 1986; Tziotziou et al. 2002) and into the transition region between the chromosphere and the corona (Gurman et al. 1982), see reviews by Lites (1992) and Bogdan (2000) and recent papers by Fludra (2001), Maltby et al. (2001), and Brynildsen et al. (2002). Part of the $3 \mathrm{~min}$ wave energy penetrates into the corona along sunspot coronal loops (Maltby et al. 1999; Brynildsen et al. 2002; De Moortel et al. 2002).

In this paper we explore a new approach to the investigations of oscillations above sunspot by focusing on the intensity variations in the opposite wings of the emission lines. A similar method has been used in studies of the $5 \mathrm{~min}$ oscillations in photospheric $\mathrm{Fe}$ I/I lines to investigate the wave properties in the solar atmosphere, see Bertello et al. (1992) and references given therein. The behaviour of opposite wings of the Na I D lines has been discussed in helioseismology investigations, see Ulrich et al. (2000). We find that the analysis of opposite line wings in the emission lines is less complicated than previous applications to other spectral lines. To our knowledge, investigations of the intensity variations in opposite wings of optically thin emission lines have not previously been presented.

Send offprint requests to: P. Maltby, e-mail: per.maltby@astro.uio.no

\section{Observations}

The focus in this paper is on spectra of nine sunspots observed in 1998, 1999, and 2000 with the Solar Ultraviolet Measurements of Emitted Radiation (SUMER; Wilhelm et al. 1995) on the Solar and Heliospheric Observatory (SOHO; Domingo et al. 1995), see Brynildsen et al. (2000) and Maltby et al. (2001). Briefly, the spectra are obtained with an exposure time of $15 \mathrm{~s}$ while the solar rotation moves the sunspot image over the narrow slit $\left(0.3^{\prime \prime} \times 120^{\prime \prime}\right)$. From consecutive exposures we extract spatial and spectral windows equal to $120^{\prime \prime} \times 2.2(1.1) \AA$ in the first (second) order of the grating. The spectral windows are centered on the transition region lines $\mathrm{N} v \lambda \lambda 1238,1242$ in the first order and Ov $\lambda 629$ in the second order of the grating. The latter spectral window includes the chromospheric Si II $\lambda 1260$ line.

\section{Results}

Based on close inspection of the spectra we find that it is easier to detect the $3 \mathrm{~min}$ oscillations above sunspots in the short wavelength ("blue") wing than in the long wavelength ("red") wing of the lines. In the following the line wings are defined as the wavelength interval that corresponds to the interval between 0.1 and 0.4 times the peak line intensity in the 20 min time-averaged line profile. Figure 1 shows the temporal variations in the relative intensities in opposite wings of the O v $\lambda 629, \mathrm{~N} v \lambda 1238$ and $\mathrm{Si}$ II $\lambda 1260$ lines, observed on 9 May 2000 in NOAA 8989. The $N \vee \lambda 1238$ and $N v \lambda 1242$ lines originate from the same muliplet and show similar characteristics, the latter line is not included in Fig. 1. Evidently, the temporal variations in relative line intensity in the blue wing, $\Delta \mathrm{I}_{\mathrm{b}} / \bar{I}_{\mathrm{b}}$, suggest that oscillations are present in $\mathrm{O} \vee \lambda 629, \mathrm{~N} \vee \lambda 1238$ and Si II $\lambda 1260$. The intensity variations in the red wing, $\Delta I_{\mathrm{r}} / \bar{I}_{\mathrm{r}}$, are 

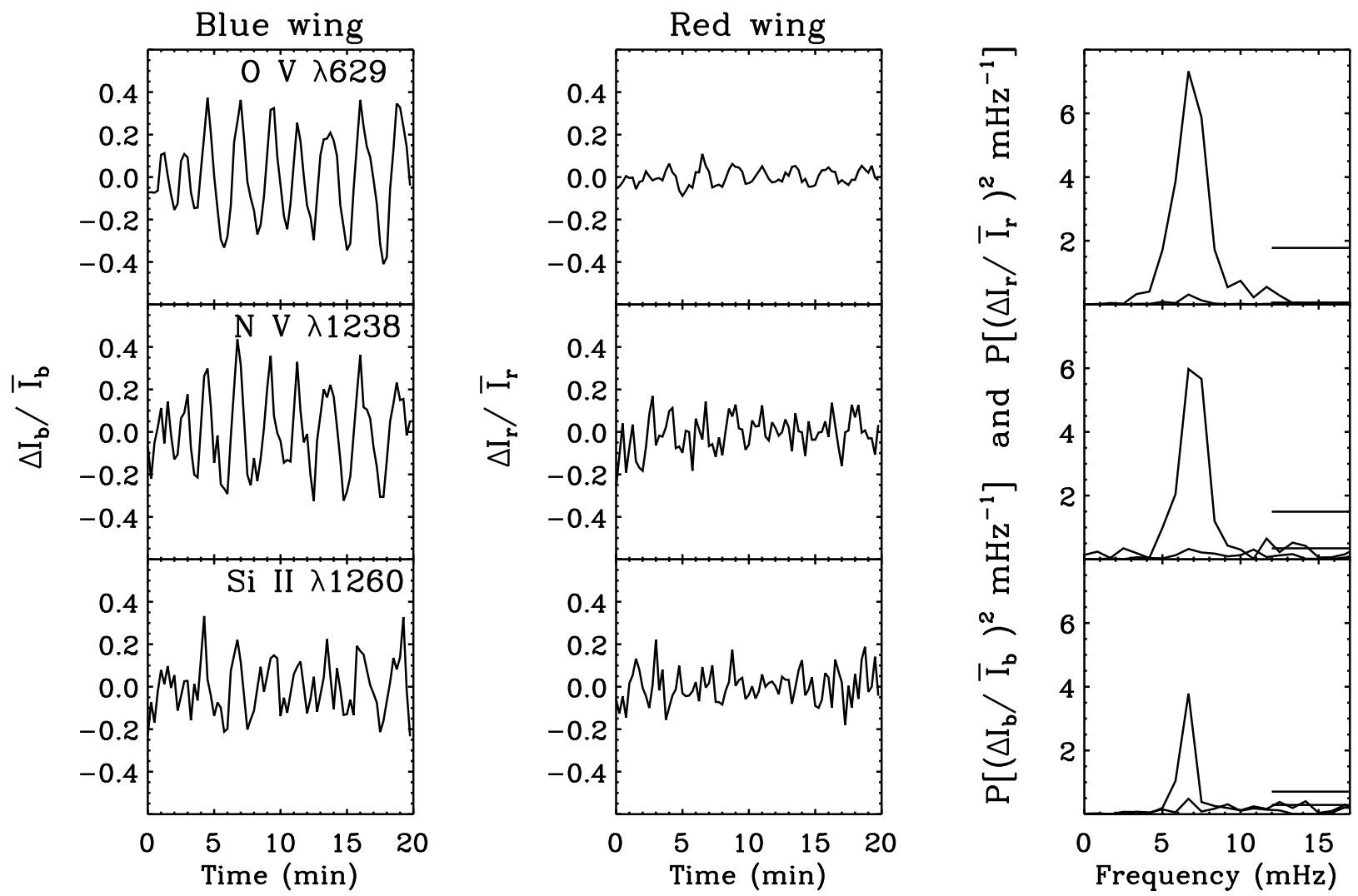

Fig. 1. Left to right: observed temporal variation in relative line intensity in the blue wing, $\Delta I_{\mathrm{b}} / \bar{I}_{\mathrm{b}}$, followed by the temporal variation in the red wing, $\Delta I_{\mathrm{r}} / \bar{I}_{\mathrm{r}}$, and the corresponding power spectra, $P\left[\left(\Delta I_{\mathrm{b}} / \bar{I}_{\mathrm{b}}\right)^{2}\right]$ (thick line) and $P\left[\left(\Delta I_{\mathrm{r}} / \bar{I}_{\mathrm{r}}\right)^{2}\right]$, for the transition region lines $\mathrm{O} \mathrm{V} \lambda 629$ and N v $\lambda 1238$ and the chromospheric Si II $\lambda 1260$ line, observed on 9 May 2000 in NOAA 8989. The horizontal lines mark three times the noise level for the blue (thick line) and red wing in the power spectra plots.

considerably smaller and the presence of oscillations is not so obvious.

The significance of the peaks in the power spectra is evaluated by determining the noise level from power spectra derived from the same set of intensity values after a randomization of the points in time. Three times the noise level is marked by horizontal lines in the power plots. Hence, only the power peak close to $6.5 \mathrm{mHz}$ is significant for $\mathrm{O} \vee \lambda 629$, $\mathrm{N} v \lambda 1238$ and $\mathrm{Si}$ II $\lambda 1260$ in Fig. 1. Comparing the power spectra at $6.5 \mathrm{mHz}$ we find that $P\left[\left(\Delta \mathrm{I}_{\mathrm{b}} / \bar{I}_{\mathrm{b}}\right)^{2}\right]$ is considerably larger than $P\left[\left(\Delta I_{\mathrm{r}} / \bar{I}_{\mathrm{r}}\right)^{2}\right]$ for the each of the lines.

Note that the power spectra in both the transition region lines, $\mathrm{O} v \lambda 629, \mathrm{~N} v \lambda 1238$ and the chromospheric line $\mathrm{Si}$ II $\lambda 1260$ show only one significant frequency, close to $6.5 \mathrm{mHz}$. As pointed out by Bogdan et al. (2002) recent observations do not support the sunspot filter theory, based on the idea of a chromospheric resonator. Whereas the observations show one dominant frequency in the power spectra, the filter theory predicts several peaks caused by resonances and equally spaced $\approx 1 \mathrm{mHz}$ in frequency, see Settele et al. (2001) and references given therein.

Figure 2 shows the temporal intensity variations in opposite wings of the Ov $\lambda 629$ line for several sunspots. The oscillations show one dominant frequency, close to $6 \mathrm{mHz}$, this value of the dominant frequency is found in most of the sunspots. The power spectra in Fig. 2 strengthen the statement made above that the oscillation amplitudes in the blue wing, $\Delta I_{\mathrm{b}} / \bar{I}_{\mathrm{b}}$, are considerably larger than the amplitudes, $\Delta I_{\mathrm{r}} / \bar{I}_{\mathrm{r}}$, in the red wing. Hence, it is easier to detect the 3 min oscillations above sunspots in the blue wing than in the red wing of the emission lines.

\section{Discussion}

Brynildsen et al. (1999a,b) have compared the observations of intensity and line-of-sight velocity in the 3 min sunspot oscillations with the predictions of the acoustic wave theory and found the observations to be compatible with upwardly propagating acoustic waves, as proposed by Gurman et al. (1982). The waves are linear in some sunspots, but show a non-linear character in others.

For an optically thin emission line formed in the chromosphere, transition region or corona the emergent intensity $I$ is determined by the integral along the line-of-sight of the product of the number density of the emitting ion, the collisional excitation rate, and the emission profile (e.g., Mariska 1992). The following calculation may be of interest. To a first approximation the line intensity, $I$, is proportional to the square of the particle density $\rho$ and the relation may be written $I / I_{0} \sim\left(\rho / \rho_{0}\right)^{2}$, where the index zero denotes the undisturbed value. Consider a one-dimensional traveling, nonlinear acoustic wave without shocks, often called a simple wave. For a polytropic gas the 

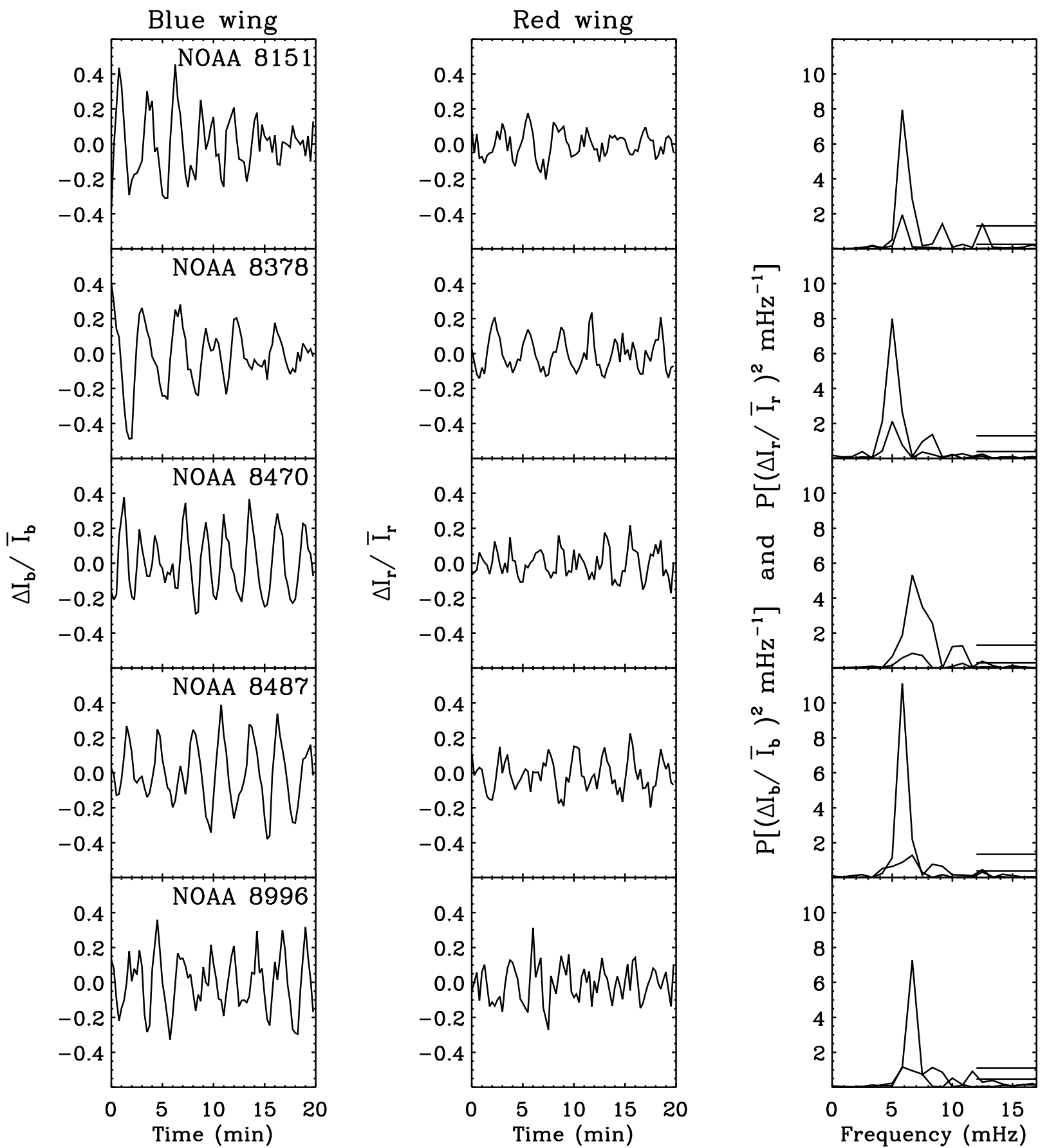

Fig. 2. Left to right: observed temporal variations in relative line intensity in the blue wing, $\Delta I_{\mathrm{b}} / \bar{I}_{\mathrm{b}}$, followed by the temporal variation in the red wing, $\Delta I_{\mathrm{r}} / \bar{I}_{\mathrm{r}}$, and the corresponding power spectra, $P\left[\left(\Delta I_{\mathrm{b}} / \bar{I}_{\mathrm{b}}\right)^{2}\right]$ (thick line) and $P\left[\left(\Delta I_{\mathrm{r}} / \bar{I}_{\mathrm{r}}\right)^{2}\right]$ for $\mathrm{OV} \lambda 629$ in several sunspots. The horizontal lines mark three times the noise level for the blue (thick line) and red wing in the power spectra plots.

relation between the particle velocity, $u$, and the density, $\rho$, is (e.g., Landau \& Lifshitz 1987):

$\rho=\rho_{0}\left[1 \pm \frac{1}{2}(\gamma-1) \frac{u}{c_{0}}\right]^{2 /(\gamma-1)}$,

where $\gamma$ is the polytropic index and $c_{0}$ is the unperturbed sound speed. Since we measure velocities away from the observer as positive, the minus sign should be selected for an upwardly propagating wave. To compare the observations with the spectral signatures of an upwardly propagating wave we must take into account the aspect angle, in this study derived from the sunspot location on the solar disk assuming vertically propagating waves.

It is the phase correlation between the density and velocity perturbations in a compressive wave that will insure that a spectral line will preferentially radiate while the plasma is being compressed, i.e., while the plasma is approaching in an upwardly propagating wave. We neglect the line-of-sight velocity originating from the displacement of the line forming region 

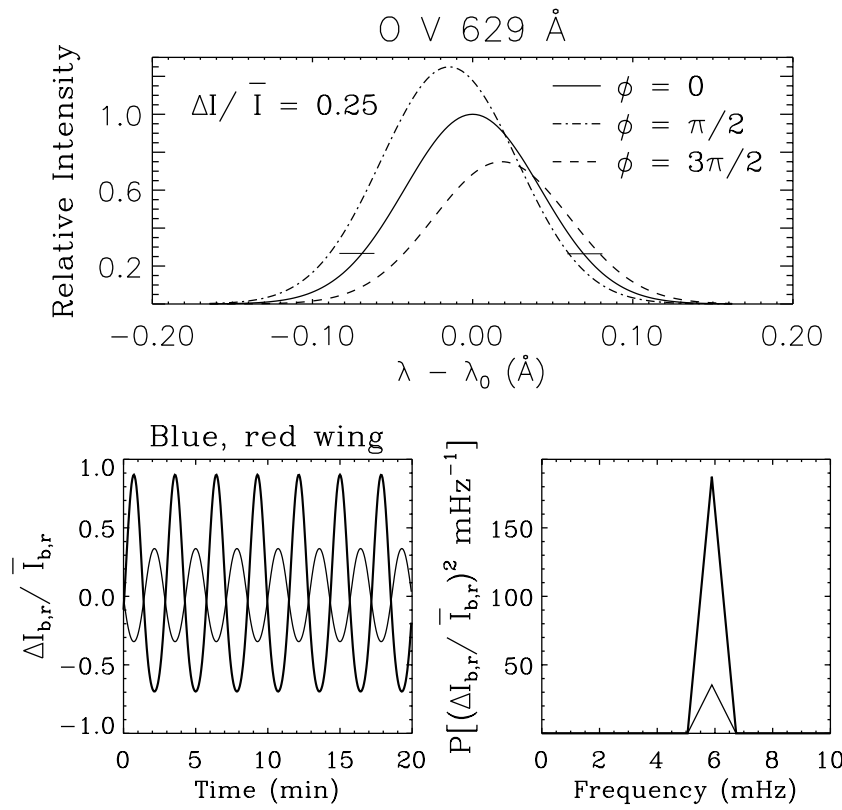

Fig. 3. Calculated Ov $\lambda 629$ line profiles, emitting from a region pervaded by an upwardly propagating, nonlinear acoustic wave with $(\Delta I / \bar{I})=\left(\Delta \rho / \rho_{0}\right)^{2}=0.25$ for three values of the phase, $\phi($ top $)$. The locations of the blue and red wings are marked with horizontal bars. The calculated temporal variations in relative line intensity in the blue, $\Delta I_{\mathrm{b}} / \bar{I}_{\mathrm{b}}$ (thick line), and red wing, $\Delta I_{\mathrm{r}} / \bar{I}_{\mathrm{r}}$, and the corresponding power spectra, $P\left[\left(\Delta I_{\mathrm{b}} / \bar{I}_{\mathrm{b}}\right)^{2}\right]$ (thick line $)$ and $P\left[\left(\Delta I_{\mathrm{r}} / \bar{I}_{\mathrm{r}}\right)^{2}\right]$ are shown in the bottom diagrams.

caused by the pulse momentum of the wave and the motions set up to restore the quasi-equilibrium of the line forming region.

For three values of the phase angle, $\phi$, Fig. 3 shows the calculated Ov $\lambda 629$ line profile. The calculation is based on the assumption that $\mathrm{O} v \lambda 629$ is emitted from a region pervaded by an upwardly propagating, nonlinear acoustic wave with $(\Delta I / \bar{I})=\left(\Delta \rho / \rho_{0}\right)^{2}=0.25$. The calculated oscillations in the opposite line wings of $\mathrm{O} v \lambda 629$ and the corresponding power spectra are also shown. The calculations show that the oscillation amplitudes in relative line intensity in the blue wing, $\Delta I_{\mathrm{b}} / \bar{I}_{\mathrm{b}}$, are considerably larger than the oscillations in the red wing, $\Delta I_{\mathrm{r}} / \bar{I}_{\mathrm{r}}$. Comparing Figs. 1 and 2 with the calculations in Fig. 3 we find that the observed oscillations in the opposite line wings appear to be compatible with upwardly propagating acoustic waves.

Figure 3 predicts that the oscillations in the blue wing should lead the oscillations in the red wing by $180^{\circ}$. The observations, on the other hand, suggest differences between $100^{\circ}$ and $160^{\circ}$. We have run some experiments where the SUMER spectral resolution and the observed line width variations are taken into account in the calculations. This brings the calculated phase difference between the line wings closer to the observed values. It is outside the scope of this paper to repeat the calculations without the assumptions made above.

Acknowledgements. SUMER is supported by DLR, CNES, NASA and the ESA Prodex programme (Swiss contribution). This study was supported by the Research Council of Norway. SOHO is a mission of international cooperation between ESA and NASA.

\section{References}

Beckers, J. M., \& Tallant, P. E. 1969, Sol. Phys., 7, 351

Bertello, L., Caccin, B., Francia, P., \& Pietropaolo, E. 1992, ApJ, 401, 768

Bogdan, T. J. 2000, Sol. Phys., 192, 373

Bogdan, T. J., Rosenthal, C. S., Carlsson, M., et al. 2002, Astron. Nachr., 323, 196

Brynildsen, N., Leifsen, T., Kjeldseth-Moe, O., Maltby, P., \& Wilhelm, K. 1999a, ApJ, 511, L121

Brynildsen, N., Kjeldseth-Moe, O., Maltby, P., \& Wilhelm, K. 1999b, ApJ, 517, L159

Brynildsen, N., Maltby, P., Fredvik, T., \& Kjeldseth-Moe, O. 2002, Sol. Phys., 207, 259

Brynildsen, N., Maltby, P., Leifsen, T., Kjeldseth-Moe, O., \& Wilhelm, K. 2000, Sol. Phys., 191, 129

De Moortel, I., Ireland, J., Hood, A. W., \& Walsh, R. W. 2002, A\&A, 387, L13

Domingo, V., Fleck, B., \& Poland, A. I. 1995, Sol. Phys., 162, 1

Fludra, A. 2001, A\&A, 368, 639

Gurman, J. B., Leibacher, J. W., Shine, R. A., Woodgate, B. E., \& Henze, W. 1982, ApJ, 253, 939

Landau, L. D., \& Lifshitz, E. M. 1987, Fluid Mechanics (Oxford, Pergamon Press), 380

Lites, B. W. 1986, ApJ, 301, 1005

Lites, B. W. 1992, in Sunspots: Theory and Observations, ed. J. H. Thomas, \& N. O. Weiss (Dordrecht, Kluwer), 261

Maltby, P., Brynildsen, N., Fredvik, T., Kjeldseth-Moe, O., \& Wilhelm, K. 1999, Sol. Phys., 190, 437

Maltby, P., Brynildsen, N., Kjeldseth-Moe, O., \& Wilhelm, K. 2001, A\&A, 373, L1

Mariska, J. T. 1992, The Solar Transition Region (Cambridge, Cambridge University Press)

Settele, A., Staude, J., \& Zhugzhda, Y. D. 2001, Sol. Phys., 202, 281

Solanki, S., Livingston, W., Muglach, K., \& Wallace, L. 1996, A\&A, 315,303

Tziotziou, K., Tsiropoula, G., \& Mein, P. 2002, A\&A, 381, 279

Ulrich, R. K., Garca, R. A., Robillot, J.-M., et al. 2000, A\&A, 364, 799

Wilhelm, K., Curdt, W., Marsch, E., et al. 1995, Sol. Phys., 162, 189 